\title{
Physiotherapie bei kolorektalen Erkrankungen des Kindes- und Jugendalters
}

\author{
N. Gürnth; A. Brock; M. Lacher \\ Klinik und Poliklinik für Kinderchirurgie, Universitätsklinikum Leipzig
}

\section{Schlüsselwörter}

Physiotherapie, Atemtherapie, Beckenboden, Stuhlinkontinenz, kolorektale Erkrankungen

\section{Zusammenfassung}

Viele Kinder mit kolorektalen Erkrankungen leiden unter chronischer Verstopfung oder Stuhlinkontinenz, deren Ursache auch in einer muskulären Dysbalance oder neuronalen Störung des Beckenbodens begründet sein kann. Therapeutische Behandlungsansätze stellen die sakrale Nervenstimulation, Biofeedback oder die Beckenbodengymnastik dar. Letztere findet ihren Ansatzpunkt im Erlernen und Verbessern der Perzeption des knöchernen Beckens und muskulären Beckenbodens. Bewusste Körperwahrnehmung bildet die Grundlage für aktives Beckenbotätsprüfungen geben Rückschlüsse auf den Tonus der Beckenbodenmuskulatur. Atemtherapeutische Maßnahmen wie auch Dehnlagerungen, aktive und passive Techniken können die Darmperistaltik und das Atemgleichgewicht positiv beeinflussen. Anschließend werden Muskelimbalancen mithilfe der gewonnenen Perzeption und Atemtechniken aktiv in den Normotonus trainiert. Grundlage hierfür bilden Mobilisations-, Koordinationsund Gleichgewichtsübungen in verschiedenen Ausgangsstellungen. Eine spaßorientierte und vertrauensvolle Therapieeinheit begünstigt den Lernerfolg und motiviert das Kind zur Durchführung des individuellen Hausübungsprogrammes. dentraining. Das Alignement und die Mobili-
Keywords

Physical therapy, respiratory therapy, pelvic floor, alignment, colorectal diseases

\section{Summary}

Many children with colorectal diseases suffer from chronic constipation or anal incontinence, most probably caused by the muscular imbalance of the pelvic floor. The stimulation of the sacral nerves, biofeedback or perineal gymnastics are options to treat the patient. The rationale for an active training of the pelvic floor is to learn and improve the perception of the osseous and muscular pelvis. In turn, alignment and mobility influence the tonus of muscular pelvic floor. Respiratory therapy influences the intestinal peristalsis, the balance of breathing, and may have relaxing effects on the patient. With the training of mobility, coordination and balance, the patient will be able to improve the muscular pelvic floor towards normotonia. A conception of enjoyable and trustful therapy appears fundamental since it is able to improve patient's outcome and motivates the child to work out with an individual exercise program at home.

The role of physical therapy in children and adolescents suffering from colorectal diseases Kinder- und Jugendmedizin 2018; 18: 34-38 Eingereicht am: 22. Juni 2017; angenommen am: 27. Juli 2017
Viele Kinder mit angeborenen kolorektalen Erkrankungen wie anorektalen Malformationen (ARM) oder dem Morbus Hirschsprung (MH) leiden unter chronischer Verstopfung oder Stuhlinkontinenz. Bei deren Therapie kommen häufig Medikamente wie Stuhlweichmacher (z. B. Lactulose, Macrogol) oder Laxanzien (z. B. Sennesblätter) zum Einsatz (4). Die Ursache einer Stuhlinkontinenz oder Verstopfung kann jedoch auch in einer muskulären Dysbalance des Beckenbodens begründet sein. Um hier therapeutisch einzugreifen, werden Therapieformen wie die sakrale Nervenstimulation oder das Biofeedback angewendet. Die Wertigkeit dieser Verfahren ist sehr umstritten (1). Um mit dem Biofeedback arbeiten zu können, ist zudem die kognitive Fähigkeit des Patienten ausschlaggebend, welche altersabhängig ist (2).

2013 wurde erstmalig über den Einsatz von physiotherapeutischen Behandlungstechniken zur Therapie von kolorektalen Funktionsstörungen berichtet (5). Ziel dieses Artikels ist es, über unsere Erfahrungen mit dieser Therapieform zu berichten mit besonderem Fokus auf die praktischen physiotherapeutischen Aspekte (6-8).

Ziele der Physiotherapie sind:

- Körperwahrnehmung

- Kräftigung

- funktionelle Balance zwischen Zwerchfell und Beckenboden

- Dehnung

- Detonisierung/Entspannung.

\section{Beginn der Behandlung}

Klinik und Poliklinik für Kinderchirurgie

Universitätsklinikum Leipzig

Liebigstraße 20a, 04103 Leipzig

Tel.: 0341 97-26400, Fax: -409

E-Mail: Martin.Lacher@medizin.uni-leipzig.de
Zunächst ist es wichtig, eine vertrauensvolle therapeutische Beziehung zum Kind und dessen Familie aufzubauen. Eine Stuhlinkontinenz ist ein sehr intimes Thema. Die 
Erkrankung ist vielen Patienten unangenehm, daher können sie oft zu Beginn noch nicht über ihre Situation und vor allem ihren Körper sprechen.

Die ersten Minuten der Therapie sollten daher für ein „warm up“ mit dem Ziel des „bondings" zwischen Patient und Thera- peut genutzt werden. Wir lassen dem Kind daher die erste Wahl über ein Therapiegerät (z. B. Trampolin, Weichbodenmatte), um über einen spaßorientierten Lernerfolg die Verbindung zum Patienten zu schaffen ( A Abb. 1a-b).
Abb. 1

Hüpfen auf dem Gymnastikball erwärmt und lockert die Muskulatur (a). Sprungvariationen auf dem Trampolin wirken aktivierend und testen vorab motorische Fähigkeiten (b).
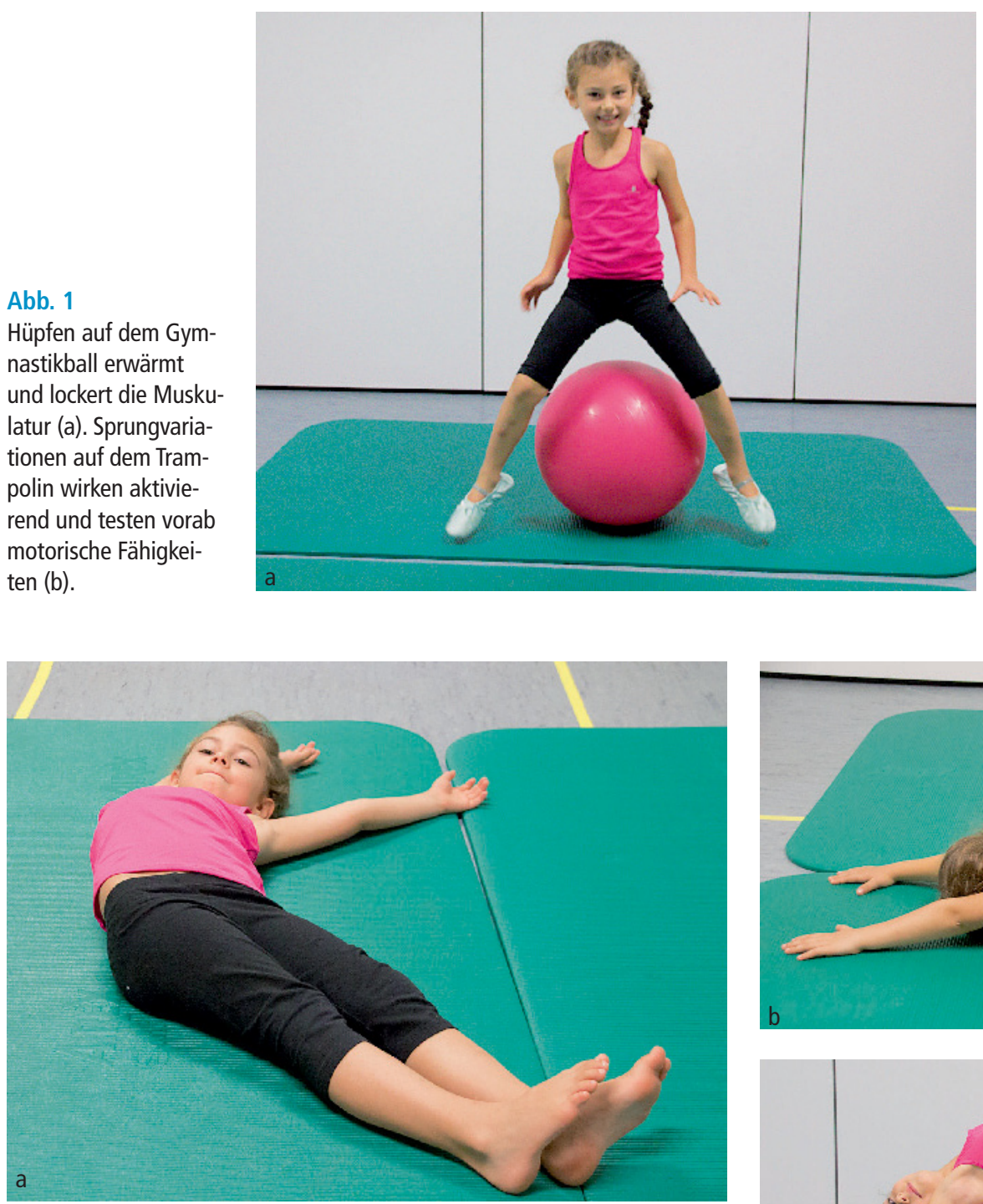

Abb. 2 "Halbmondlage": Durch vertiefte Einatmung wird die offene Flanke gedehnt (a).

"Rutsche": Streckung der Wirbelsäule in Kombination mit vertiefter Bauchatmung lockert den unteren Rücken und bewirkt anschließend einen ruhigen Atemrhythmus (b).

„Eslein streck'dich": Die Öffnung des Brustkorbes führt anschließend zur vertieften Inspiration, senkt die Atemfrequenz und wirkt beruhigend (c).

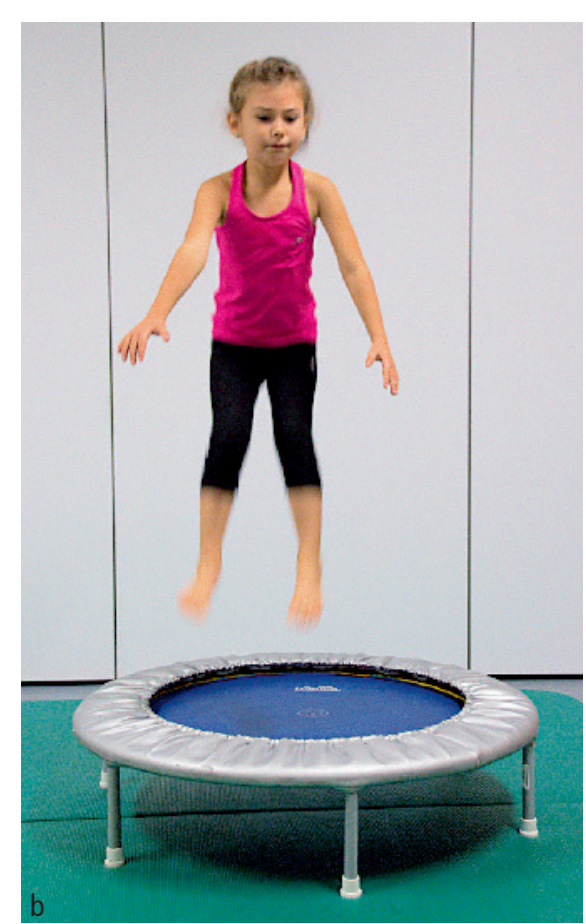

\section{Körperwahrnehmung}

Ist der Behandlungseinstieg geglückt, stellt das nächste Ziel eine verbesserte Körperwahrnehmung dar. Nur aufgrund der Perzeption eines Patienten ist später ein gezieltes Training der Beckenbodenaktivität mög-
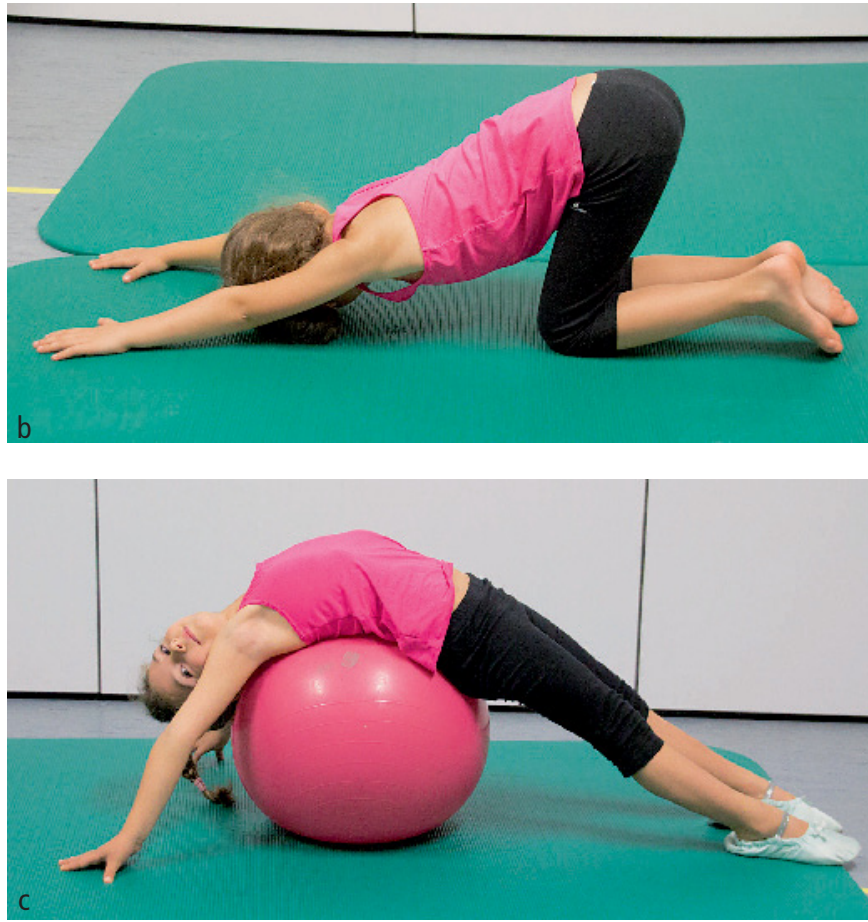


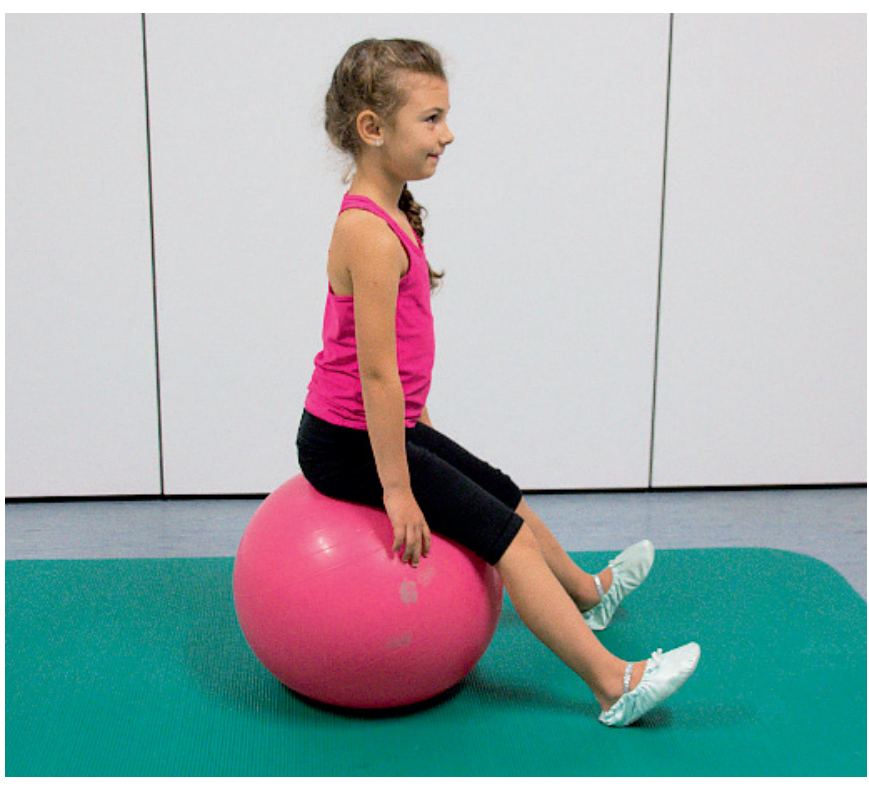

Abb. 3

Aufrechter Sitz auf dem Gymnastikball mit Vor- und Rückwärtsrollen - eingeleitet über die Füße

lich. Ein Training der „Sinne“ entspricht demnach auch einem Training der Muskulatur. Eine willkürliche muskuläre Ansteuerung kann nur geschehen, wenn das Kind auch ein Gespür und eine Vorstellung von der Lage der Beckenbodenmuskulatur hat. Über das Alignement der Körperachsen und die Mobilität des knöchernen Beckens sind schon erste Rückschlüsse auf den Tonus der Beckenbodenmuskulatur sowie auch andere Muskelgruppen des Rumpfes zu schließen. Aus der Überführung des Normotonus in einen Hyper- oder Hypotonus spezifischer Muskelgruppen resultiert eine Veränderung des Spannungsgrades aller beteiligter Synergisten und Antagonisten der komplexen Muskelkette. Folgende Fragen sollte der Therapeut sich stellen:

1. Welche Muskelimbalancen bestehen aufgrund der Körperhaltung?

2. Welche Auswirkungen haben diese auf den Beckenboden? (direkt oder indirekt)

3. Wird dadurch die Balance zwischen Zwerchfell und Beckenbodenmuskulatur gestört?

\section{Atemtherapie}

Einen besonderen Stellenwert bei der Behandlung von kolorektalen Erkrankungen hat auch die Atemtherapie. Allein durch jede vertiefte In- und Exspiration wird die Darmperistaltik angeregt (3). Man spricht dabei auch von „Bauchmassage“. Grundlegend muss dafür ein muskuläres Gleichgewicht beider Diaphragmata sowie eines normotonen Muskelzylinders gewährleistet sein. Unter einem Muskelzylinder versteht man dabei das Zusammenspiel der Bauchmuskulatur (M. obliquus externus et internus abdominis, $\mathrm{M}$. transversus abdominis, M. rectus abdominis) und den kurzen Muskeln der Wirbelsäule (Mm. multifidi, $\mathrm{Mm}$. rotatores breves et longi), welche zentrierend auf die Wirbelsäule wirken. Das funktionelle Zusammenspiel zwischen Zwerchfell, Beckenbodenmuskulatur und der abdominellen Muskulatur beeinflusst die intraabdominellen Druckverhältnisse und somit die Kontinenz. Die Atemtherapie kann dabei auf verschiedene Arten (mit und ohne Hilfsmittel, aktiv oder passiv, in verschiedensten Ausgangsstellungen etc.) gestaltet werden. Da der Fokus der Therapie auf der Perzeption liegt, bieten sich verschiedenste Dehnlagerungen an, um Einfluss auf das Atemgleichgewicht zu nehmen. Die Übungen dämpfen die Sympathikusaktivität, wirken somit beruhigend und fördern die vertiefte Einatmung. Dadurch lösen sich Anspannungen und der Atemfluss kommt ins Gleichgewicht. Zudem verändert sich auf neuromuskulärer Ebene die Spannung der Skelettmuskulatur, es kommt zur Relaxation ( $\triangleright$ Abb. 2a-c).
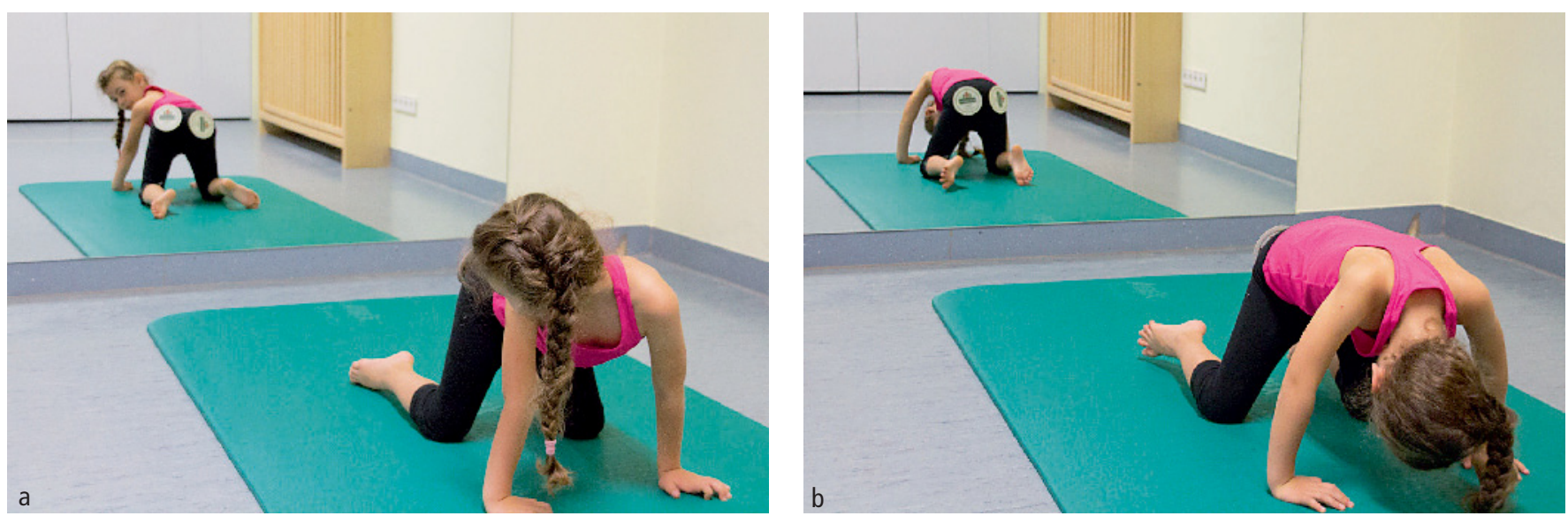

Abb. 4 "Scheinwerfer": spielerisches Entdecken des eigenes Körpers und damit verbundene Mobilisation im Vierfüßlerstand (a); „Scheinwerfer”: Aufgrund der stabilen Ausgangsstellung ist das kreative Arbeiten mit dem gesamten Körper gut möglich (b). 
Abb. 5

Greifen mit den Füßen im Einbeinstand (a); Einbeinstand auf unebener Fläche und zusätzliches Balancieren eines Reifens (sehr anspruchsvoll für jüngere Kinder) (b)
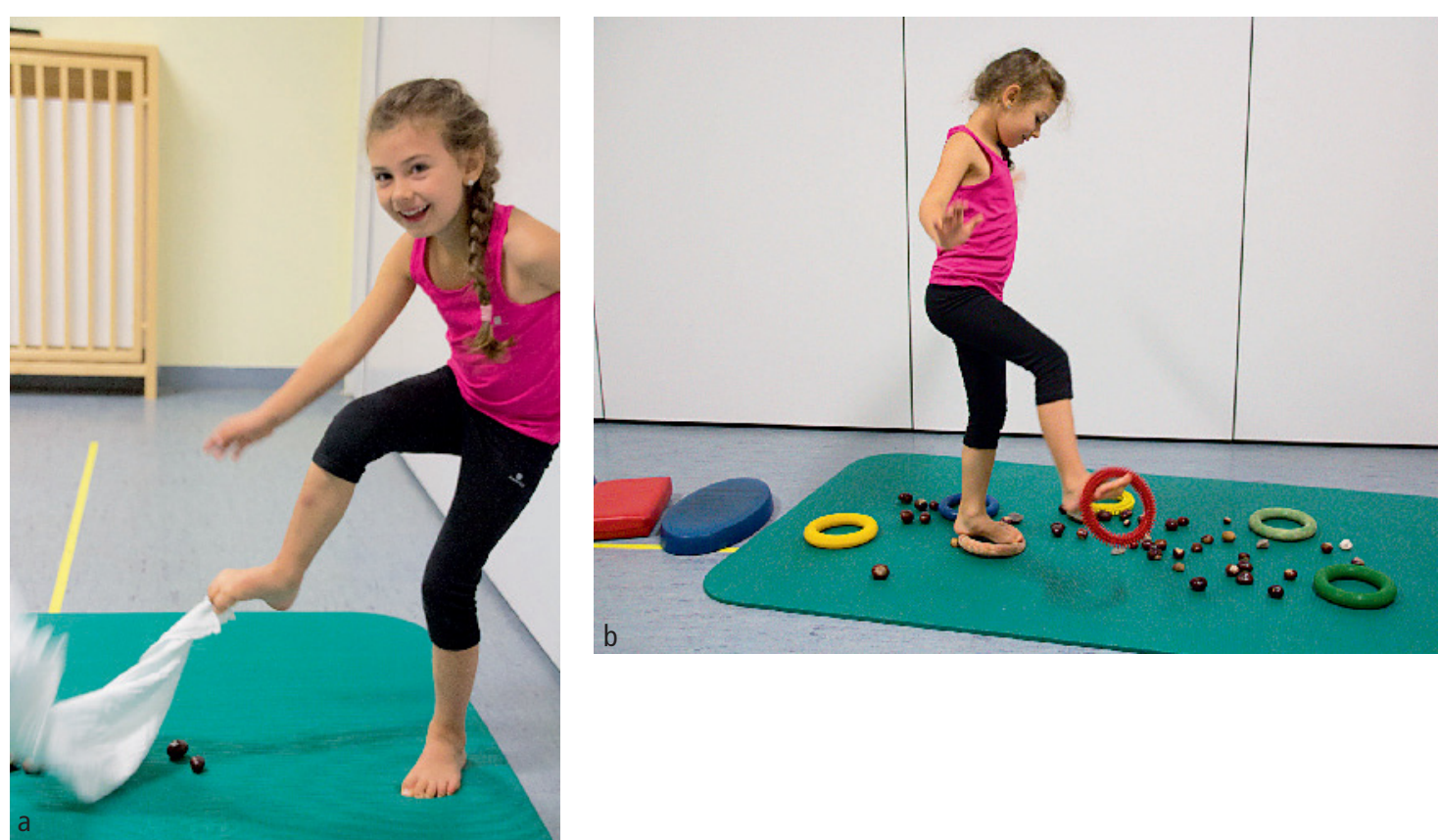

\section{Tonisierung und Detonisierung}

Sind nun globale Muster erkannt, sollten die neu gewonnene Perzeption und Atemtechnik Anwendung in komplexeren Abläufen finden. In den meisten Fällen ist das Problem nicht ausschließlich das Lösen oder Anspannen der Beckenbodenmuskulatur, sondern zum Teil sind beide Aufgaben in ihrer Aktivität „verschoben“. Daher sollte der Fokus der therapeutischen Behandlung sowohl auf der Tonisierung als auch Detonisierung liegen. Spezialisierte Beckenbodentherapeuten bedienen sich einer Vielzahl von therapeutischen Mitteln, $\mathrm{zu}$ denen unter anderem der Gymnasitkball gehört, der insbesondere bei Kindern mit kolorektalen Erkrankungen viele Vorteile hat. Für Kinder steht der Spaßfaktor immer im Vordergrund. Durch Rollen, Hüpfen, Liegen auf dem Ball oder andere Übungen bekommt der Patient einen großen Input an Reizen und Informationen über die Stellung des knöchernen Beckens. Bei guter Perzeption erhält er auch ein Gefühl für den Spannungsgrad der Beckenbodenmuskulatur, ohne dass der Therapeut manuell therapeutisch tätig werden muss. Der Gymnastikball eignet sich besonders für die Verbesserung von Mobilitätseinschränkungen, tonisierende und detonisierende Aufgabenstellungen, zur Erlernung
Abb. 6

Langsitz mit aufgestützten Händen: Heran- und Wegrollen eines Gegenstandes führt zur Spannungsänderung der Beckenbodenmuskulatur.

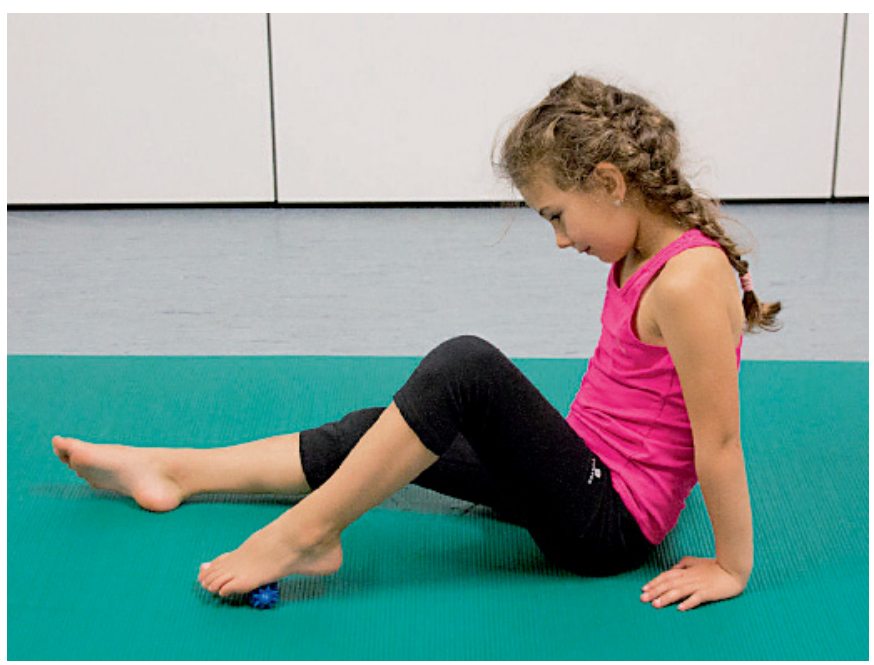

des peripheren Atemantriebs und somit zur Verinnerlichung von Atemtechniken. Je nach gewünschtem Schwierigkeitsgrad kann schon die erste intramuskuläre Koordinationsübung erfolgen.

Beim „freien Üben“ (ohne Halten des Therapeuten) trainieren die Kinder ihre Balance und damit auch ihre Perzeption $(\triangle A b b .3)$

Eine sehr komplexe, dafür aber für Kinder besonders geeignete Übung stellt der "Scheinwerfer" dar ( Abb. 4a-b). Bei diesem Training geht es im direkten Sinne um das „Entdecken“ und Wecken der Neugier am eigenen Körper. Indirekt wird durch diese Übung die Mobilität der Wirbelsäule und des knöchernen Beckens wahrgenommen sowie die Muskelaktivität der Adduktorengruppe durch Gewichtsverlagerung beim Blick um die Körperachse. Ferner erfolgt auch eine verstärkte Flankenatmung durch Dehnung bzw. Lateralflexion der Wirbelsäule.

\section{Gleichgewichts- und Greifübungen}

Viele Übungen lassen sich leicht im häuslichen Umfeld umsetzen. Hierbei kann eine 


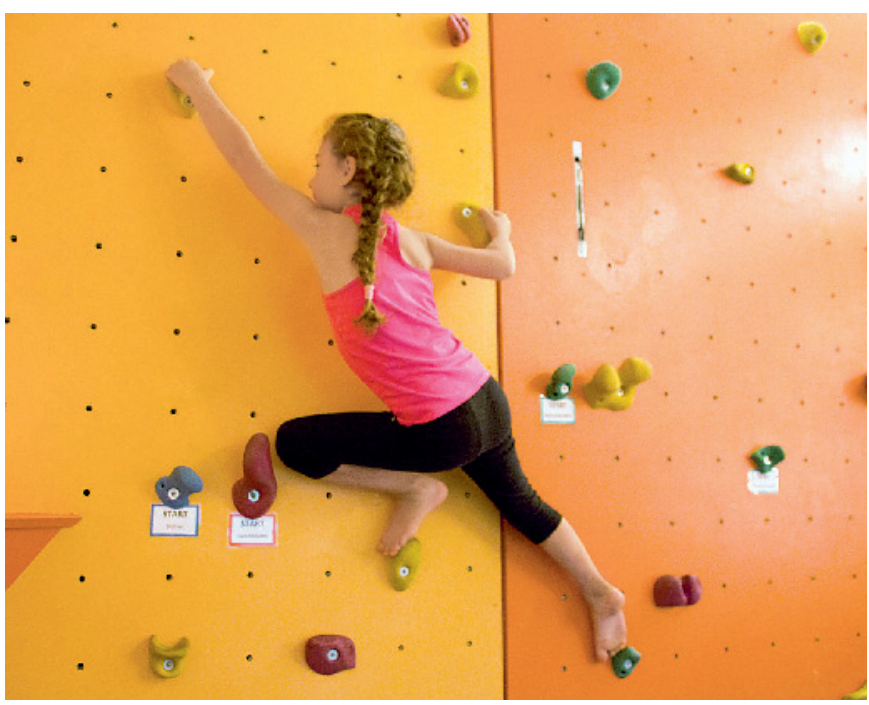

Abb. 7

Klettern trainiert die Perzeption und aktiviert die gesamte Beinund Rumpfmuskulatur.
Übung zum Einsatz kommen, die „Greifen mit den Füßen" genannt wird $(\triangleright$ Abb. 5a-b). Allein der Einbeinstand stellt eine Herausforderung für das Gleichgewichtssystem der Kinder dar. Über plantare Reflexpunkte im Rückfußbereich kommt es zu einer reflektorischen Wirkung der Becken- und Darmorgane. Wenn die Zehen beim Abrollvorgang nun weitest möglich abduziert auf dem Boden positioniert werden, aktiviert sich zudem die abdominelle Muskulatur (vgl. Bobath-Konzept). Zusätzlich führt das Spielbein mittels einer koordinativen Greifbewegung der Zehen nach einem Gegenstand zur Aktivierung der Fußmuskulatur. Über die bereits erwähnten Muskelketten erfährt der Patient sowohl eine Tonuserhöhung der gesamten Beinmuskulatur als auch eine Stärkung der Beckenbodenmuskulatur, da diese reflektorisch eine Spannungsveränderung erfährt. Eine leichtere Ausgangsstellung mit gleichem Effekt zeigt sich im Langsitz. Das Heran- und Wegrollen eines Balles oder eines ähnlichen Gegenstandes beschreibt ebenfalls den oben genannten Effekt und führt zu einer Aktivitätssteigerung des Beckenbodens ( $\triangleright$ Abb. 6).

\section{Umfeld und Häufigkeit der Übungen}

Es reicht nicht aus, einmal wöchentlich die Physiotherapiepraxis zu besuchen. Vor allem im häuslichen Umfeld müssen die Übungen regelmäßig durchgeführt werden. Daher sollten Kinder und Familien vom Therapeuten motiviert werden, zu Hause ein individuelles Trainingsprogramm durchzuführen. Mit jeder Behandlungseinheit erweitert sich das Übungsrepertoire des Patienten, sodass anfänglich koordinativ schwerere Übungen nun leichter und grundlegende Techniken verinnerlicht werden. Bei fortgeschrittenen Patienten kann es sinnvoll sein, die erlernten Techniken mit einer Sportart zu verknüpfen. Besonders eignet sich hierfür das Klettern, da alle oben genannten Techniken und Effekte bei dieser Sportart benötigt und trainiert werden ( $\triangleright$ Abb. 7).

Grundsätzlich sollte die Therapie für das Kind und die Familie Spaß und Neugier wecken. Die Behandlung darf daher keine lästige Pflicht, sondern sollte Teil einer attraktiven Freizeitgestaltung sein. Mit dem Besuch der Physiotherapiepraxis sollten somit Lust und Vorfreude auf eine spannende Stunde verbunden sein, um über eine maximale Compliance das bestmögliche Ergebnis für das Kind zu erzielen.

\section{Fazit für die Praxis}

Bei der physiotherapeutischen Arbeit mit inkontinenten Kindern spielt die Verbesserung der Körperwahrnehmung eine zentrale Rolle. Über erlernte Perzeption gelingt eine Aktivierung der Beckenbodenmuskulatur. Die Anwendung von verschiedenen therapeutischen Hilfsmitteln und Atemtherapie nimmt einen wesentlichen Einfluss auf den Muskeltonus des Beckenbodens und Imbalancen können somit ausgeglichen werden.

\section{Literatur}

1. Brazzelli M, Griffiths PV, Cody JD, Tappin D. Behavioural and cognitive interventions with or without other treatments for the management of faecal incontinence in children. Cochrane Database Syst Rev 2011 Dec 7; (12): CD002240. doi: 10.1002/14651858.CD002240.pub4. Review

2. Hüter-Becker A, Dölken M. Physiotherapie in der Neurologie, 3. Aufl. Stuttgart, New York: Thieme Verlag 2010.

3. Rutte R, Sturm S. Atemtherapie, 2. Aufl. Berlin, Heidelberg, New York: Springer Verlag 2010.

4. Santos-Jasso KA, Arredondo-García JL, Maza-Vallejos J, Lezama-Del Valle P. Effectiveness of senna vs polyethylene glycol as laxative therapy in children with constipation related to anorectal malformation. J Pediatr Surg 2017; 52: 84-88.

5. van Engelenburg-van Lonkhuyzen ML, Bols EM, Benninga MA et al. The effect of pelvic physiotherapy on reduction of functional constipation in children: design of a multicentre randomised controlled trial. BMC Pediatr 2013; 13: 112.

6. van Engelenburg-van Lonkhuyzen ML, Bols EM, Benninga MA, Verwijs WA, de Bie RA. Bladder and bowel dysfunctions in 1748 children referred to pelvic physiotherapy: clinical characteristics and locomotor problems in primary, secondary, and tertiary healthcare settings. Eur J Pediatr 2017; 176: 207-216.

7. van Engelenburg-van Lonkhuyzen ML, Bols EM, Benninga MA et al. Effectiveness of pelvic physiotherapy in children with functional constipation compared with standard medical care. Gastroenterology 2017; 152: 82-91.

8. van Summeren J, Dekker J, Berger M. Pelvic physiotherapy in children with functional constipation: promising but more research Needed. Gastroenterology 2017; 152: 2080-2081. 